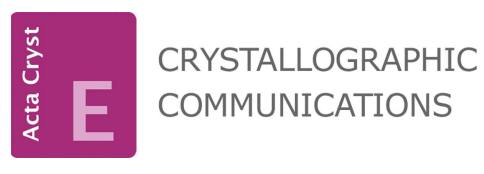

ISSN 2056-9890

Received 29 December 202

Accepted 27 February 2022

Edited by C. Schulzke, Universität Greifswald, Germany

Keywords: crystal structure; acetohydrazide; Hirshfeld surface analysis; lanthanide(III) ion; terbium(III).

Supporting information: this article has supporting information at journals.iucr.org/e
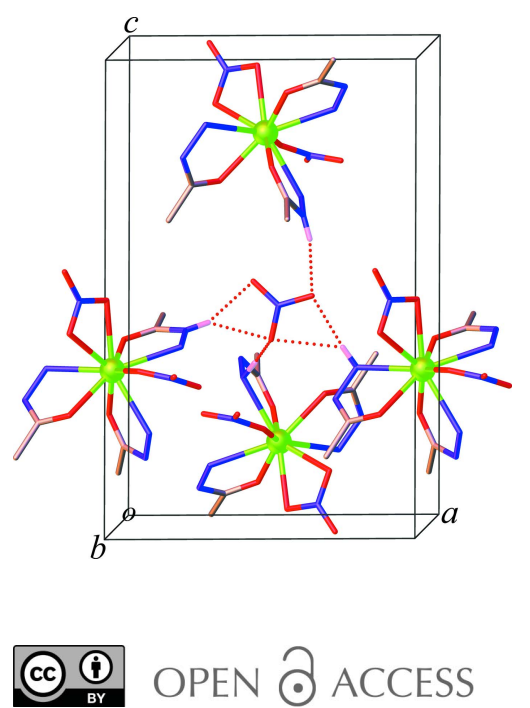

Published under a CC BY 4.0 licence

\section{Crystal structure and Hirshfeld surface analysis of tris(acetohydrazide- $\left.\kappa^{2} N, O\right)($ nitrato- $\kappa O)($ nitrato- $\left.\kappa^{2} O, O^{\prime}\right)$ terbium(III) nitrate}

\author{
Chatphorn Theppitak, ${ }^{a}$ Sakchai Laksee ${ }^{\mathrm{b}}$ and Kittipong Chainok $^{\mathrm{a} *}$
}

${ }^{\mathbf{a}}$ Thammasat University Research Unit in Multifunctional Crystalline Materials and Applications (TU-McMa), Faculty of Science and Technology, Thammasat University, Khlong Luang, Pathum Thani, 12121, Thailand, and ${ }^{\mathbf{b}}$ Nuclear Technology Research and Development Center, Thailand Institute of Nuclear Technology (Public Organization), Ongkharak, Nakon Nayok, 26120, Thailand. *Correspondence e-mail: kc@tu.ac.th

In the title lanthanide(III) compound, $\left[\mathrm{Tb}\left(\mathrm{NO}_{3}\right)_{2}\left(\mathrm{C}_{2} \mathrm{H}_{6} \mathrm{~N}_{2} \mathrm{O}\right)_{3}\right] \mathrm{NO}_{3}$, the asymmetric unit contains one $\mathrm{Tb}^{3+}$ ion, three acetohydrazide $\left(\mathrm{C}_{2} \mathrm{H}_{6} \mathrm{~N}_{2} \mathrm{O}\right)$ ligands, two coordinated nitrate anions, and an isolated nitrate anion. $\mathrm{The}^{3 \mathrm{~Tb}^{3+}}$ ion is in a ninefold coordinated distorted tricapped trigonal-prismatic geometry formed by three oxygen atoms and three nitrogen atoms from three different acetohydrazide ligands and three oxygen atoms from two nitrate anions. In the crystal, the complex molecules and the non-coordinated nitrate anions are assembled into a three-dimensional supramolecular architecture through extensive $\mathrm{N}-\mathrm{H} \cdots \mathrm{O}$ hydrogen-bonding interactions between the amine $\mathrm{NH}$ groups of the acetohydrazide ligands and the nitrate oxygen atoms. Hirshfeld surface analysis was performed to aid in the visualization of intermolecular contacts.

\section{Chemical context}

Over the past two decades, there has been increasing interest in the construction of new lanthanide-based coordination compounds, not only because of their structural diversity but also because of their fascinating potential applications in luminescence, magnetism, adsorption, and similar areas (Roy et al., 2014; Cui et al., 2018; Kuwamura et al., 2021). It is well known that lanthanide(III) ions have a high affinity for and prefer binding to hard donor atoms. Thus, organic ligands with oxygen donor atoms such as aromatic polycarboxylic acids have been used extensively for the formation of these coordination materials (Janicki et al., 2017) whereas organohydrazide ligands have received far less attention. Accordingly, a ConQuest search of the Cambridge Structural Database (CSD, Version 5.42, September 2021 update; Bruno et al., 2002; Groom et al., 2016) reveals only 23 entries for hydrazidecontaining lanthanide complexes. Among them, 15 lanthanide coordination complexes have recently been reported by our groups. Some of these complexes exhibited a high $\mathrm{CO}_{2}$ uptake ability at high pressure (Theppitak et al., 2021a), and have shown great potential as luminescent sensors for acetone and the $\mathrm{Co}^{2+}$ ion with good recyclability (Theppitak et al., 2021b). In this work, we present the molecular structure of a new terbium(III) complex, $\left[\mathrm{Tb}\left(\mathrm{C}_{2} \mathrm{H}_{6} \mathrm{~N}_{2} \mathrm{O}\right)_{3}\left(\mathrm{NO}_{3}\right)_{2}\right] \mathrm{NO}_{3} \quad$ (1), synthesized with acetohydrazide $\left(\mathrm{C}_{2} \mathrm{H}_{6} \mathrm{~N}_{2} \mathrm{O}\right)$ as the organic ligand. In addition, a Hirshfeld surface analysis and twodimensional fingerprint plots were used to quantify the intermolecular contacts in the crystal structure. 


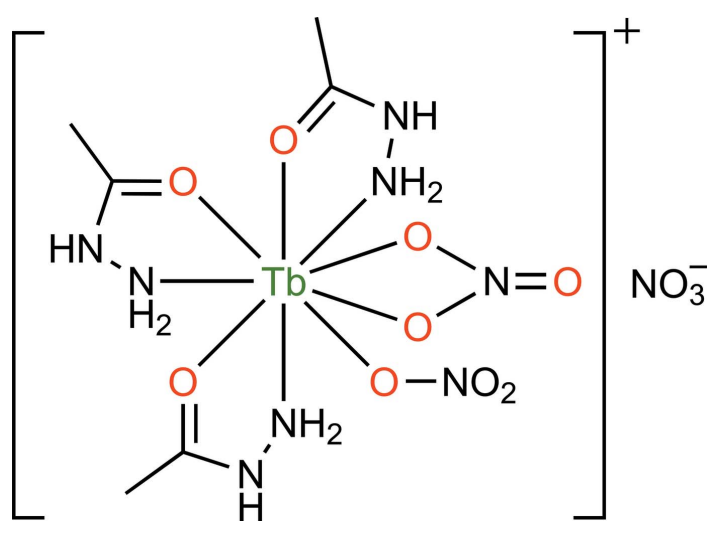

\section{Structural commentary}

The molecular structure of $\mathbf{1}$ is shown in Fig. 1. The asymmetric unit contains one $\mathrm{Tb}^{3+}$ ion, three acetohydrazide ligands, two coordinated nitrate anions, and a non-coordinated nitrate counter-anion. The $\mathrm{Tb}^{3+}$ ion is ninefold coordinated $\left(\mathrm{TbN}_{3} \mathrm{O}_{6}\right)$ by three nitrogen atoms and three oxygen atoms from three different acetohydrazide ligands, two oxygen atoms from one chelate nitrate anion, and one oxygen atom from another nitrate anion. As can be seen in Fig. 2, the coordination polyhedron of the $\mathrm{Tb}^{3+}$ ion is best described as having a distorted tricapped trigonal-prismatic geometry, wherein the $\mathrm{N} 3, \mathrm{~N} 5, \mathrm{O} 1, \mathrm{O} 3, \mathrm{O} 4$, and $\mathrm{O} 7$ atoms form a trigonal prism, while the $\mathrm{N} 1, \mathrm{O} 2$, and $\mathrm{O} 5$ atoms act as caps. $\mathrm{The} \mathrm{Tb}-\mathrm{O}$ bond lengths of 2.353 (2)-2.496 (2) $\AA$ are slightly shorter than the $\mathrm{Tb}-\mathrm{N}$ bond lengths $[2.553$ (2)-2.586 (2) $\AA$ ]. The bond angles around the central $\mathrm{Tb}^{3+}$ ion fall into the range of $50.93(7)-$ $150.97(7)^{\circ}$. These values are comparable to those reported for

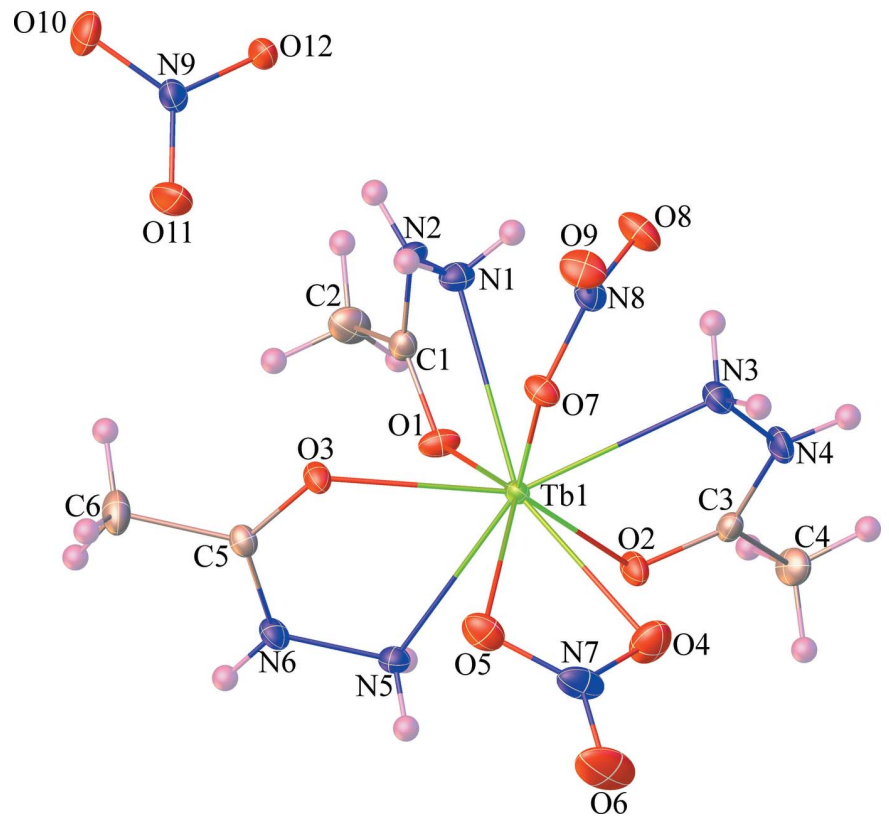

Figure 1

Molecular structure of $\mathbf{1}$, showing the atom-labeling scheme. Displacement ellipsoids are drawn at the $50 \%$ probability level.
Table 1

Hydrogen-bond geometry $\left(\AA,^{\circ}\right)$.

\begin{tabular}{|c|c|c|c|c|}
\hline$D-\mathrm{H} \cdots A$ & $D-\mathrm{H}$ & $\mathrm{H} \cdots A$ & $D \cdots A$ & $D-\mathrm{H} \cdots A$ \\
\hline $\mathrm{N} 1-\mathrm{H} 1 A \cdots \mathrm{O} 8$ & $0.84(2)$ & $2.37(2)$ & $2.950(3)$ & $126(2)$ \\
\hline $\mathrm{N} 1-\mathrm{H} 1 B \cdots \mathrm{O} 10^{\mathrm{i}}$ & $0.85(2)$ & $2.36(2)$ & $3.136(3)$ & $153(3)$ \\
\hline $\mathrm{N} 2-\mathrm{H} 2 \ldots \mathrm{O} 11$ & $0.85(2)$ & $2.69(3)$ & 3.070 (3) & $109(2)$ \\
\hline $\mathrm{N} 2-\mathrm{H} 2 \cdots \mathrm{O} 12$ & $0.85(2)$ & $2.09(2)$ & $2.891(2)$ & $156(3)$ \\
\hline $\mathrm{N} 3-\mathrm{H} 3 A \cdots \mathrm{O} 8$ & $0.87(2)$ & $2.46(3)$ & $2.866(3)$ & $110(2)$ \\
\hline $\mathrm{N} 3-\mathrm{H} 3 A \cdots \mathrm{O} 9^{\mathrm{ii}}$ & $0.87(2)$ & $2.33(2)$ & $3.146(3)$ & $157(2)$ \\
\hline $\mathrm{N} 3-\mathrm{H} 3 B \cdots \mathrm{O} 6^{\mathrm{iii}}$ & $0.85(2)$ & $2.25(2)$ & $3.089(3)$ & $168(3)$ \\
\hline $\mathrm{N} 4-\mathrm{H} 4 \cdots \mathrm{O} 10^{\mathrm{iv}}$ & $0.87(2)$ & $2.34(2)$ & $3.102(3)$ & $147(3)$ \\
\hline $\mathrm{N} 4-\mathrm{H} 4 \ldots \mathrm{O} 11^{\mathrm{iv}}$ & $0.87(2)$ & $2.17(2)$ & $2.984(3)$ & $156(3)$ \\
\hline $\mathrm{N} 5-\mathrm{H} 5 A \cdots \mathrm{O} 10^{\mathrm{v}}$ & $0.86(2)$ & $2.58(2)$ & $3.176(3)$ & $128(2)$ \\
\hline $\mathrm{N} 5-\mathrm{H} 5 A \cdots \mathrm{O} 12^{\mathrm{v}}$ & $0.86(2)$ & $2.11(2)$ & $2.964(2)$ & $173(3)$ \\
\hline $\mathrm{N} 5-\mathrm{H} 5 B \cdots \mathrm{O}^{\mathrm{vi}}$ & $0.85(2)$ & $2.51(2)$ & 3.211 (2) & $140(2)$ \\
\hline $\mathrm{N} 6-\mathrm{H} 6 \cdots \mathrm{O} 7^{\mathrm{vi}}$ & $0.85(2)$ & $2.17(2)$ & $2.999(2)$ & $166(2)$ \\
\hline $\mathrm{N} 6-\mathrm{H} 6 \cdots \mathrm{O} 10^{\mathrm{v}}$ & $0.85(2)$ & $2.74(2)$ & $3.170(3)$ & $114(2)$ \\
\hline
\end{tabular}

other ninefold-coordinated $\mathrm{Tb}^{3+}$ compounds containing oxygen/nitrogen-donor ligands such as $\left[\mathrm{Tb}\left(\mathrm{C}_{17} \mathrm{H}_{13} \mathrm{~N}_{3}\right)\left(\mathrm{NO}_{3}\right)_{2^{-}}\right.$ (DMSO)] $\mathrm{CH}_{3} \mathrm{OH}$ (VUKNEW, Chen et al., 2015) and $\left[\mathrm{Tb}\left(\mathrm{C}_{13} \mathrm{H}_{22} \mathrm{~N}_{3}\right)\left(\mathrm{NO}_{3}\right)_{3}\right] \cdot \mathrm{MeCN}$ (SEZTOJ, Long et al., 2018).

\section{Supramolecular features}

Extensive hydrogen-bonding interactions involving the three components of the hydrazide group of the acetohydrazide ligand and the coordinated and non-coordinated nitrate ions contribute to the stabilization of the supramolecular structure of 1 (Table 1; the $\mathrm{N}-\mathrm{H}$ distances are all fixed with $\mathrm{N}-\mathrm{H}=$ $0.86 \pm 0.02 \AA$ ). A closer inspection of the structure reveals that the $\left[\mathrm{Tb}\left(\mathrm{C}_{2} \mathrm{H}_{6} \mathrm{~N}_{2} \mathrm{O}\right)_{3}\left(\mathrm{NO}_{3}\right)_{2}\right]^{+}$complex molecules form centrosymmetric dimers via pairs of symmetry-related $\mathrm{N} 3-$

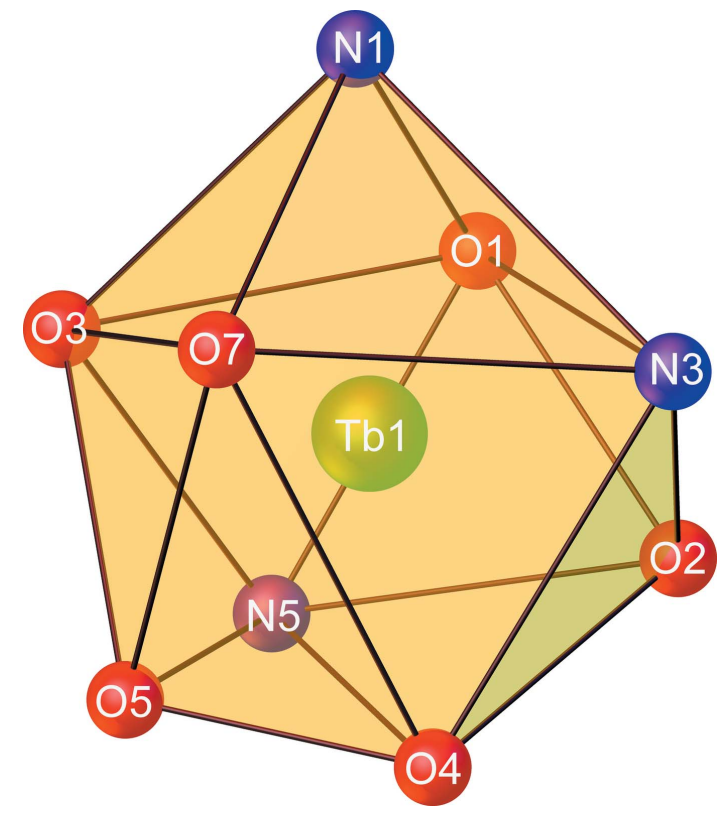

Figure 2

View of the distorted tricapped trigonal-prismatic coordination geometry of the central $\mathrm{Tb}^{3+}$ atom in $\mathbf{1}$. 


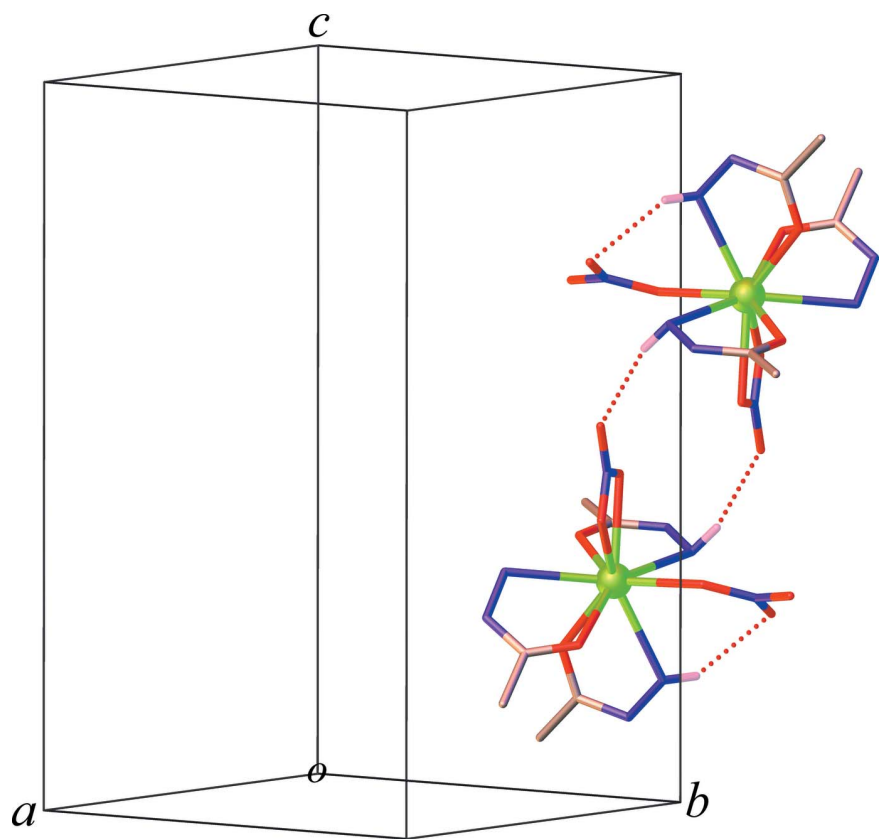

Figure 3

Dimer formation through $\mathrm{N}-\mathrm{H}$. . O hydrogen bonds (dashed lines) in $\mathbf{1}$ (hydrogen atoms, except those forming hydrogen bonds, are omitted for clarity).

$\mathrm{H} 3 B \cdots \mathrm{O} 6$ hydrogen bonds involving the amine $\mathrm{NH}$ group of the acetohydrazide ligand and the coordinated nitrate oxygen atom, Fig. 3. Notably, the amine NH donor and the coordinated nitrate oxygen acceptor is also involved in an intramolecular $\mathrm{N} 1-\mathrm{H} 1 A \cdots \mathrm{O} 8$ hydrogen bond. The dimers are further held together through an intermolecular N3$\mathrm{H} 3 A \cdots \mathrm{O} 9$ hydrogen bond between the amine $\mathrm{NH}$ and the coordinated nitrate oxygen (O9), resulting in the formation of a two-dimensional supramolecular layer that propagates in the [100] direction, Fig. 4. Ultimately, adjacent layers are connected into a three-dimensional supramolecular architecture via the other two complementary $\mathrm{N}-\mathrm{H} \cdots \mathrm{O}$

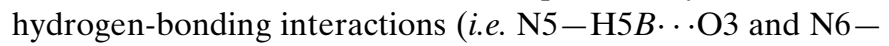

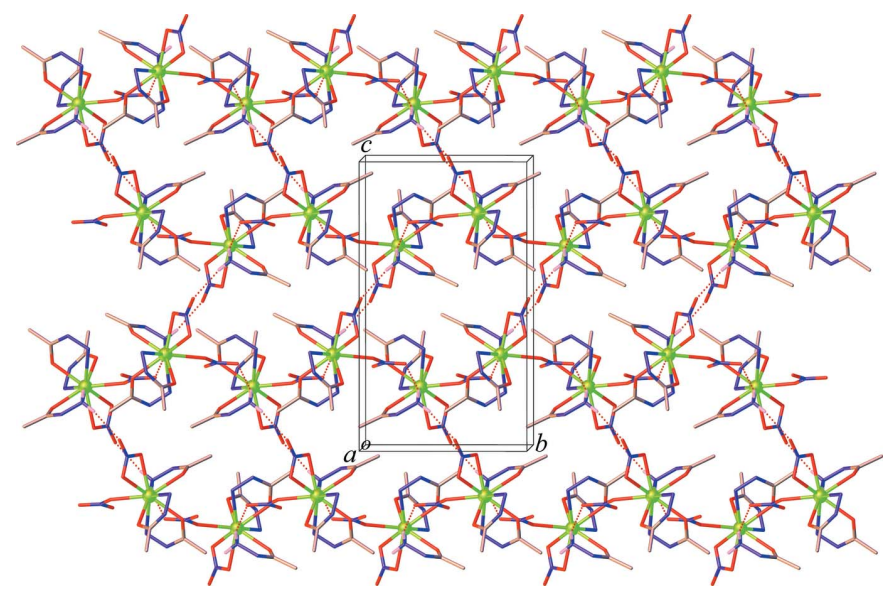

Figure 4

The two-dimensional hydrogen bonded layer in $\mathbf{1}$ (hydrogen atoms, except those forming hydrogen bonds, are omitted for clarity).

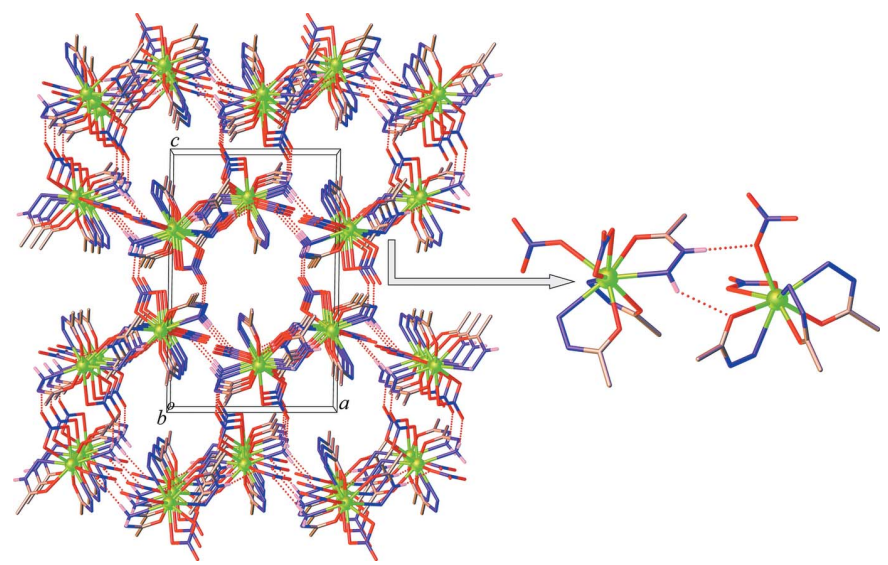

Figure 5

The three-dimensional hydrogen-bonded network in $\mathbf{1}$ (hydrogen atoms, except those forming hydrogen bonds, are omitted for clarity).

H6 ...07) occurring between the acetohydrazide ligands and the coordinated nitrate ions, Fig. 5. In addition, the noncoordinated nitrate anion is located in cavities along the $b$ axis and serves as the acceptor site for six $\mathrm{N}-\mathrm{H} \cdots \mathrm{O}$ hydrogenbonding interactions (i.e. $\mathrm{N} 1-\mathrm{H} 1 B \cdots \mathrm{O} 10, \mathrm{~N} 2-\mathrm{H} 2 \cdots \mathrm{O} 12$, $\mathrm{N} 4-\mathrm{H} 4 \cdots \mathrm{O} 10, \mathrm{~N} 4-\mathrm{H} 4 \cdots \mathrm{O} 11, \mathrm{~N} 5-\mathrm{H} 5 A \cdots \mathrm{O} 10$, and N5H5A...O12) as shown in Fig. 6.

\section{Hirshfeld surface analysis}

The Hirshfeld surface analysis (McKinnon et al., 2007) and the associated two-dimensional fingerprint plot generation (Spackman \& McKinnon, 2002) were carried out using Crys-

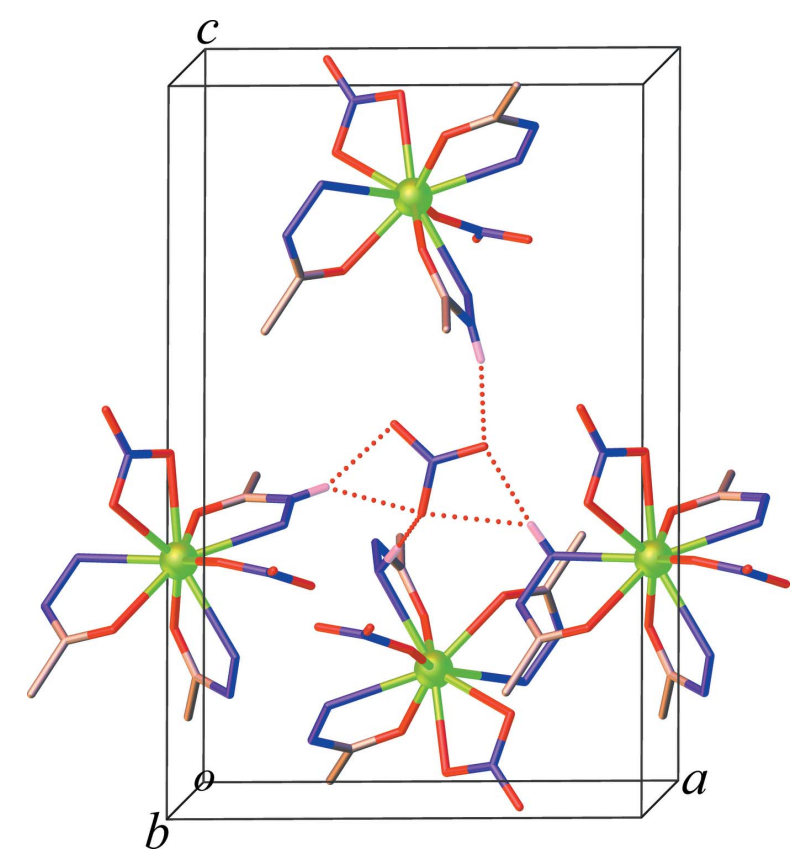

Figure 6

View of $\mathbf{1}$ approximately along the $b$-axis direction, showing the $\mathrm{N}-$ $\mathrm{H} \cdots \mathrm{O}$ hydrogen-bonding interactions involving the non-coordinated nitrate ion and the complex molecules (hydrogen atoms, except those forming hydrogen bonds, are omitted for clarity). 

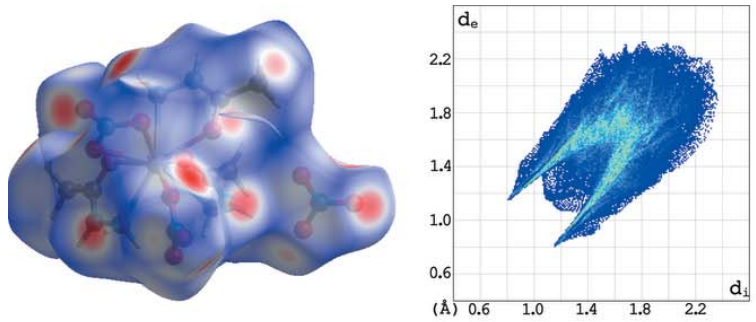

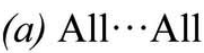
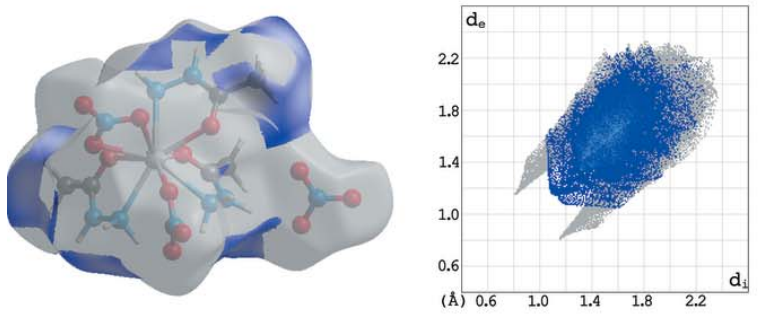

(c) $\mathrm{H} \cdots \mathrm{H}(22.8 \%)$
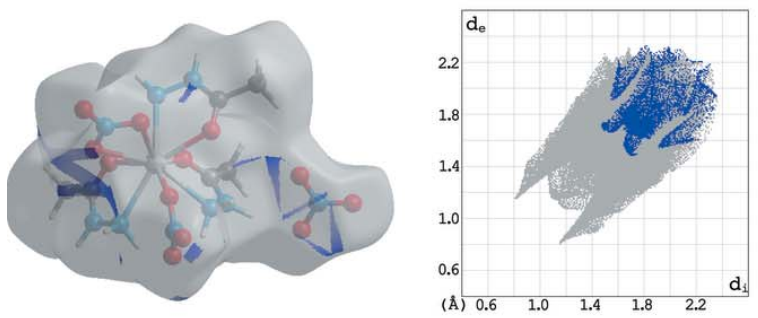

(e) $\mathrm{O} \cdots \mathrm{O}(3.7 \%)$
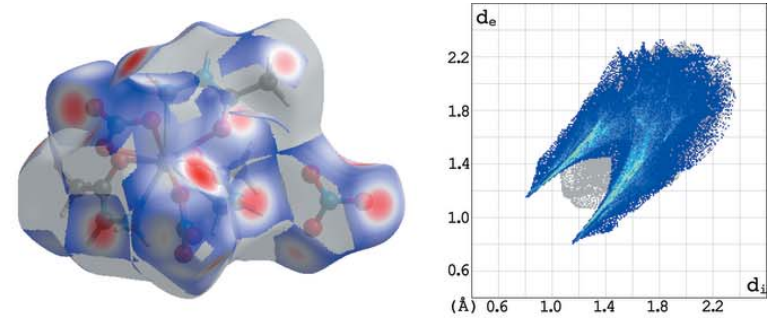

(b) $\mathrm{H} \cdots \mathrm{O} / \mathrm{O} \cdots \mathrm{H}(62.8 \%)$
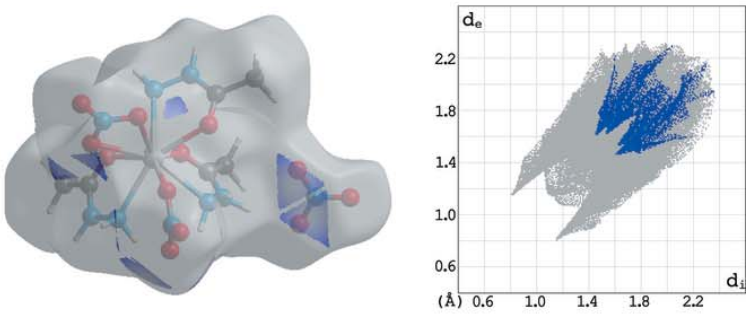

(d) $\mathrm{O} \cdots \mathrm{N} / \mathrm{N} \cdots \mathrm{O}(4.9 \%)$
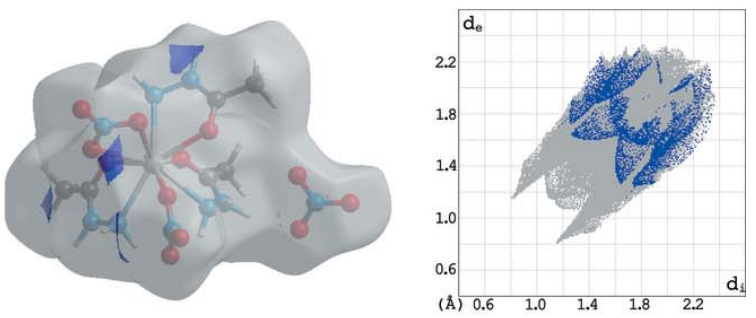

(f) $\mathrm{H} \cdots \mathrm{N} / \mathrm{N} \cdots \mathrm{H}(3.0 \%)$

Figure 7

Two-dimensional fingerprint plots of $\mathbf{1}$, showing $(a)$ all interactions, and those delineated into $(b) \mathrm{H} \cdots \mathrm{O} / \mathrm{O} \cdots \mathrm{H},(c) \mathrm{H} \cdots \mathrm{H},(d) \mathrm{N} \cdots \mathrm{O} / \mathrm{O} \cdots \mathrm{N},(e) \mathrm{O} \cdots \mathrm{O}$, and $(f) \mathrm{H} \cdots \mathrm{N} / \mathrm{N} \cdots \mathrm{H}$ contacts $\left[d_{\mathrm{e}}\right.$ and $d_{\mathrm{i}}$ represent the distances from a point on the Hirshfeld surface to the nearest atoms outside (external) and inside (internal) the surface, respectively].

talExplorer17 (Turner et al., 2017) in order to quantify the nature of the intermolecular interactions present in the crystal structure, and the results are shown in Figs. 7 and 8. The most significant contributions to the $d_{\text {norm }}$ surfaces are $\mathrm{H} \cdots \mathrm{O} /$

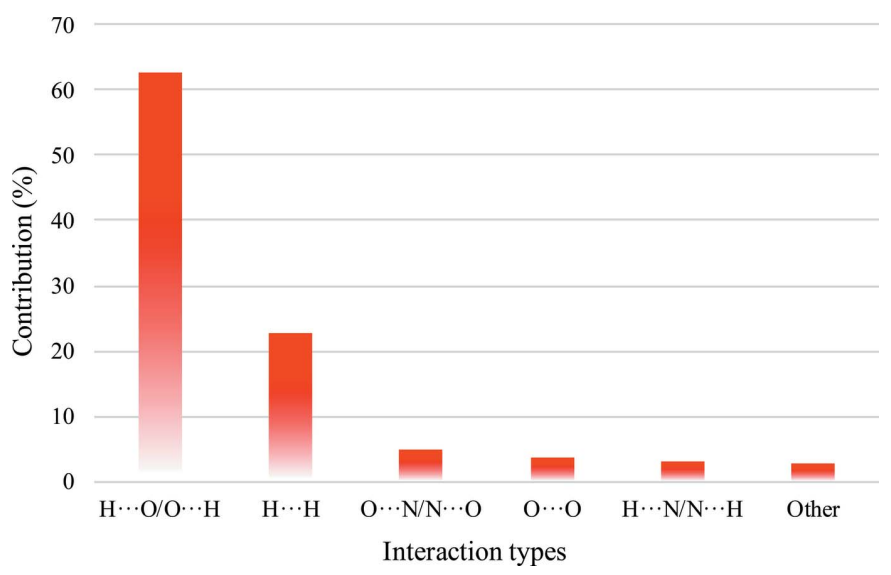

Figure 8

Quantitative results of different intermolecular contacts contributing to the Hirshfeld surface of $\mathbf{1}$.
$\mathrm{O} \cdots \mathrm{H}$ contacts (i.e. $\mathrm{N}-\mathrm{H} \cdots \mathrm{O}$ hydrogen bonds), contributing $62.8 \%$ to the overall crystal packing of the title compound. The $\mathrm{H} \cdots \mathrm{H}$ contacts (representing van der Waals interactions) with a $22.8 \%$ contribution play a minor role in the stabilization of the crystal packing. All other $\mathrm{N} \cdots \mathrm{O} / \mathrm{O} \cdots \mathrm{N}, \mathrm{O} \cdots \mathrm{O}$ and $\mathrm{H} \cdots \mathrm{N} / \mathrm{N} \cdots \mathrm{H}$ contacts make only negligible contributions to the Hirshfeld surface.

\section{Database survey}

A ConQuest search of the Cambridge Structural Database (CSD, Version 5.42, September 2021 update; Bruno et al., 2002; Groom et al., 2016) for the structures of lanthanide complexes with acetohydrazide ligands gave ten hits, viz. Er [CECLEB (Pangani et al., 1983), CECLEB10 (Agre et al., 1984)], Dy [CECLIF (Pangani et al., 1983), CECLIF10 (Pangani, Agre et al., 1984)], Ho [CECLOL (Pangani et al., 1983), CECLOL10 (Pangani, Agre et al., 1984)], Pr (CUWFAB; Pangani, Machhoshvili et al., 1984), Gd (FOYGIM; Brandão et al., 2020), and Sm [ISNHSM (Zinner et al., 1979), QITBIH (Theppitak et al., 2018)]. In all of these complexes, the acetohydrazide ligand adopts a $\mu_{2}-\kappa^{1}: \kappa^{1}$ bidentate chelating coordination mode to 
bind the lanthanide(III) ion and the amine $\mathrm{NH}$ moiety of the acetohydrazide ligand can act as a donor site for intermolecular hydrogen-bonding interactions, similar to that of the title compound.

\section{Synthesis and crystallization}

A mixture of $\mathrm{Tb}\left(\mathrm{NO}_{3}\right)_{3} \cdot 6 \mathrm{H}_{2} \mathrm{O}(45.3 \mathrm{mg}, 0.1 \mathrm{mmol})$, acetohydrazide (14.8 mg, $0.2 \mathrm{mmol}$ ), and isopropyl alcohol (4 ml) was sealed in a $15 \mathrm{ml}$ Teflon-lined steel autoclave and heated at $373 \mathrm{~K}$ for $24 \mathrm{~h}$. The mixture was cooled to room temperature and colorless block-shaped crystals of the title compound (1) were obtained in $87 \%$ yield $\left(39.3 \mathrm{mg}\right.$, based on $\mathrm{Tb}^{3+}$ source). Analysis calculated (\%) for $\mathrm{C}_{6} \mathrm{H}_{18} \mathrm{~N}_{9} \mathrm{O}_{12} \mathrm{~Tb}$ : C 12.71; $\mathrm{H} 3.20 ; \mathrm{N}$ $22.23 \%$. Found: C 12.44; H 3.96; N 21.89\%.

\section{Refinement}

Crystal data, data collection and structure refinement details are summarized in Table 2. All hydrogen atoms were located in difference-Fourier maps. All carbon-bound hydrogen atoms were placed in calculated positions and refined using a ridingmodel approximation with $\mathrm{C}-\mathrm{H}=0.96 \AA$ and $U_{\text {iso }}(\mathrm{H})=$ $1.5 U_{\text {eq }}(\mathrm{C})$. All nitrogen-bound hydrogen atoms were refined with a fixed distance $\mathrm{N}-\mathrm{H}=0.86 \pm 0.02 \AA$.

\section{Funding information}

The authors gratefully acknowledge the financial support provided by the Thailand Institute of Nuclear Technology (Public Organization), through its program of TINT to University (grant to KC). This study was also partially supported by the Thammasat University Research Unit in Multifunctional Crystalline Materials and Applications (TUMcMa). CT would like to acknowledge a Graduate Development Scholarship 2020, National Research Council of Thailand (contract No. 15/2563).

\section{References}

Agre, V. M., Pangani, V. S. \& Trunov, V. K. (1984). Koord. Khim. 10, 120-128

Brandão, S. G., Ribeiro, M. A., Perrella, R. V., de Sousa Filho, P. C. \& Luz, P. P. (2020). J. Rare Earths, 38, 642-648.

Bruker (2016). APEX3, SAINT and SADABS. Bruker AXS Inc., Madison, Wisconsin, USA.

Bruno, I. J., Cole, J. C., Edgington, P. R., Kessler, M., Macrae, C. F., McCabe, P., Pearson, J. \& Taylor, R. (2002). Acta Cryst. B58, 389397.

Chen, P., Zhang, M., Sun, W., Li, H., Zhao, L. \& Yan, P. (2015). CrystEngComm, 17, 5066-5073.

Cui, Y., Zhang, J., He, H. \& Qian, G. (2018). Chem. Soc. Rev. 47, 5740-5785.

Dolomanov, O. V., Bourhis, L. J., Gildea, R. J., Howard, J. A. K. \& Puschmann, H. (2009). J. Appl. Cryst. 42, 339-341.

Groom, C. R., Bruno, I. J., Lightfoot, M. P. \& Ward, S. C. (2016). Acta Cryst. B72, 171-179.

Janicki, R., Mondry, A. \& Starynowicz, P. (2017). Coord. Chem. Rev. 340, 98-133.

Kuwamura, N. \& Konno, T. (2021). Inorg. Chem. Front. 8, 2634649.
Table 2

Experimental details.

\begin{tabular}{|c|c|}
\hline \multicolumn{2}{|l|}{ Crystal data } \\
\hline Chemical formula & {$\left[\mathrm{Tb}\left(\mathrm{NO}_{3}\right)_{2}\left(\mathrm{C}_{2} \mathrm{H}_{6} \mathrm{~N}_{2} \mathrm{O}\right)_{3}\right] \mathrm{NO}_{3}$} \\
\hline$M_{\mathrm{r}}$ & 567.21 \\
\hline Crystal system, space group & Monoclinic, $P 2_{1} / n$ \\
\hline Temperature $(\mathrm{K})$ & 296 \\
\hline$a, b, c(\AA)$ & $10.9076(3), 9.7786(3), 16.8578(5)$ \\
\hline$\beta\left(^{\circ}\right)$ & $90.791(1)$ \\
\hline$V\left(\AA^{3}\right)$ & $1797.90(9)$ \\
\hline$Z$ & 4 \\
\hline Radiation type & Мo $K \alpha$ \\
\hline$\mu\left(\mathrm{mm}^{-1}\right)$ & 4.02 \\
\hline Crystal size $(\mathrm{mm})$ & $0.28 \times 0.21 \times 0.2$ \\
\hline \multicolumn{2}{|l|}{ Data collection } \\
\hline Diffractometer & Bruker D8 QUEST CMOS \\
\hline Absorption correction & $\begin{array}{l}\text { Multi-scan ( } S A D A B S ; \text { Bruker, } \\
\text { 2016) }\end{array}$ \\
\hline$T_{\min }, T_{\max }$ & $0.471,0.747$ \\
\hline $\begin{array}{l}\text { No. of measured, independent and } \\
\text { observed }[I>2 \sigma(I)] \text { reflections }\end{array}$ & $47511,6876,5752$ \\
\hline$R_{\text {int }}$ & 0.034 \\
\hline$(\sin \theta / \lambda)_{\max }\left(\AA^{-1}\right)$ & 0.770 \\
\hline \multicolumn{2}{|l|}{ Refinement } \\
\hline$R\left[F^{2}>2 \sigma\left(F^{2}\right)\right], w R\left(F^{2}\right), S$ & $0.027,0.044,1.08$ \\
\hline No. of reflections & 6876 \\
\hline No. of parameters & 293 \\
\hline No. of restraints & 9 \\
\hline H-atom treatment & $\begin{array}{l}\mathrm{H} \text { atoms treated by a mixture of } \\
\text { independent and constrained } \\
\text { refinement }\end{array}$ \\
\hline$\Delta \rho_{\max }, \Delta \rho_{\min }\left(\mathrm{e} \AA^{-3}\right)$ & $1.12,-1.13$ \\
\hline
\end{tabular}

Computer programs: APEX3 and SAINT (Bruker, 2016), SHELXT (Sheldrick, 2015a), SHELXL (Sheldrick, 2015b), and OLEX2 (Dolomanov et al., 2009).

Long, J., Lyubov, D. M., Mahrova, T. V., Cherkasov, A. V., Fukin, G. K., Guari, Y., Larionova, J. \& Trifonov, A. A. (2018). Dalton Trans. 47, 5153-5156.

McKinnon, J. J., Jayatilaka, D. \& Spackman, M. A. (2007). Chem. Commun. pp. 3814-3816.

Pangani, V. S., Agre, V. M. \& Trunov, V. K. (1983). Zh. Neorg. Khim. 28, 2136-2137.

Pangani, V. S., Agre, V. M., Trunov, V. K. \& Machkhoshvili, R. I. (1984). Koord. Khim. 10, 1128-1131.

Pangani, V. S., Machhoshvili, R. I., Agre, V. M., Trunov, V. K. \& Shchelokov, R. N. (1984). Inorg. Chim. Acta, 94, 79.

Roy, S., Chakraborty, A. \& Maji, T. P. (2014). Coord. Chem. Rev. 273274, 139-164.

Sheldrick, G. M. (2015a). Acta Cryst. A71, 3-8.

Sheldrick, G. M. (2015b). Acta Cryst. C71, 3-8.

Spackman, M. A. \& McKinnon, J. J. (2002). CrystEngComm, 4, 378392.

Theppitak, C., Jiajaroen, S., Chongboriboon, N., Chanthee, S., Kielar, F., Dungkaew, W., Sukwattanasinitt, M. \& Chainok, K. (2021b). Molecules, 26, 4428.

Theppitak, C., Kielar, F. \& Chainok, K. (2018). Acta Cryst. E74, 16911694.

Theppitak, C., Kielar, F., Dungkaew, W., Sukwattanasinitt, M., Kangkaew, L., Sahasithiwat, S., Zenno, H., Hayami, S. \& Chainok, K. (2021a). RSC Adv. 11, 24709-24721.

Turner, M. J., Mckinnon, J. J., Wolff, S. K., Grimwood, D. J., Spackman, P. R., Jayatilaka, D. \& Spackman, M. A. (2017). CrystalExplorer17. The University of Western Australia.

Zinner, L. B., Crotty, D. E., Anderson, T. J. \& Glick, M. D. (1979). Inorg. Chem. 18, 2045-2048. 


\section{supporting information}

Acta Cryst. (2022). E78, 354-358 [https://doi.org/10.1107/S2056989022002298]

\section{Crystal structure and Hirshfeld surface analysis of tris(acetohydrazide- $\kappa^{2} \mathrm{~N}, \mathrm{O}$ ) $($ nitrato- $\kappa O)\left(\right.$ nitrato- $\left.\kappa^{2} O, O^{\prime}\right)$ terbium(III) nitrate}

\section{Chatphorn Theppitak, Sakchai Laksee and Kittipong Chainok}

Computing details

Data collection: APEX3 (Bruker, 2016); cell refinement: SAINT (Bruker, 2016); data reduction: SAINT (Bruker, 2016); program(s) used to solve structure: ShelXT (Sheldrick, 2015a); program(s) used to refine structure: SHELXL (Sheldrick, 2015b); molecular graphics: OLEX2 (Dolomanov et al., 2009); software used to prepare material for publication: OLEX2 (Dolomanov et al., 2009).

Tris(acetohydrazide- $\left.\kappa^{2} \mathrm{~N}, O\right)($ nitrato- $\kappa O)\left(\right.$ nitrato- $\left.\kappa^{2} O, O^{\prime}\right)$ terbium(III) nitrate]

Crystal data

$\left[\mathrm{Tb}\left(\mathrm{NO}_{3}\right)_{2}\left(\mathrm{C}_{2} \mathrm{H}_{6} \mathrm{~N}_{2} \mathrm{O}\right)_{3}\right] \mathrm{NO}_{3}$

$M_{r}=567.21$

Monoclinic, $P 2{ }_{1} / n$

$a=10.9076(3) \AA$

$b=9.7786(3) \AA$

$c=16.8578(5) \AA$

$\beta=90.791(1)^{\circ}$

$V=1797.90(9) \AA^{3}$

$Z=4$

\section{Data collection}

Bruker D8 QUEST CMOS diffractometer

Radiation source: sealed x-ray tube, Mo

Graphite monochromator

Detector resolution: 7.39 pixels $\mathrm{mm}^{-1}$

$\omega$ and $\varphi$ scans

Absorption correction: multi-scan

(SADABS; Bruker, 2016)

$T_{\min }=0.471, T_{\max }=0.747$

\section{Refinement}

Refinement on $F^{2}$

Least-squares matrix: full

$R\left[F^{2}>2 \sigma\left(F^{2}\right)\right]=0.027$

$w R\left(F^{2}\right)=0.044$

$S=1.08$

6876 reflections

293 parameters

9 restraints

Hydrogen site location: mixed
$F(000)=1112$

$D_{\mathrm{x}}=2.096 \mathrm{Mg} \mathrm{m}^{-3}$

Mo $K \alpha$ radiation, $\lambda=0.71073 \AA$

Cell parameters from 9937 reflections

$\theta=3.0-33.1^{\circ}$

$\mu=4.01 \mathrm{~mm}^{-1}$

$T=296 \mathrm{~K}$

Block, colourless

$0.28 \times 0.21 \times 0.2 \mathrm{~mm}$

47511 measured reflections

6876 independent reflections

5752 reflections with $I>2 \sigma(I)$

$R_{\text {int }}=0.034$

$\theta_{\max }=33.2^{\circ}, \theta_{\min }=2.8^{\circ}$

$h=-16 \rightarrow 14$

$k=-15 \rightarrow 14$

$l=-25 \rightarrow 25$

$\mathrm{H}$ atoms treated by a mixture of independent

and constrained refinement

$w=1 /\left[\sigma^{2}\left(F_{\mathrm{o}}^{2}\right)+(0.0127 P)^{2}+1.6017 P\right]$

where $P=\left(F_{\mathrm{o}}^{2}+2 F_{\mathrm{c}}^{2}\right) / 3$

$(\Delta / \sigma)_{\max }=0.003$

$\Delta \rho_{\max }=1.12 \mathrm{e} \AA^{-3}$

$\Delta \rho_{\min }=-1.13$ e $\AA^{-3}$ 
Extinction correction: SHELXL2018/3

(Sheldrick, 2015b),

$\mathrm{Fc}^{*}=\mathrm{kFc}\left[1+0.001 \times \mathrm{Fc}^{2} \lambda^{3} / \sin (2 \theta)\right]^{-1 / 4}$

Extinction coefficient: 0.00248 (11)

\section{Special details}

Geometry. All esds (except the esd in the dihedral angle between two 1.s. planes) are estimated using the full covariance matrix. The cell esds are taken into account individually in the estimation of esds in distances, angles and torsion angles; correlations between esds in cell parameters are only used when they are defined by crystal symmetry. An approximate (isotropic) treatment of cell esds is used for estimating esds involving l.s. planes.

Fractional atomic coordinates and isotropic or equivalent isotropic displacement parameters $\left(\AA^{2}\right)$

\begin{tabular}{|c|c|c|c|c|}
\hline & $x$ & $y$ & $z$ & $U_{\text {iso }} * / U_{\text {eq }}$ \\
\hline Tb1 & $0.53793(2)$ & $0.69267(2)$ & $0.81234(2)$ & $0.01794(3)$ \\
\hline $\mathrm{O} 1$ & $0.53302(15)$ & $0.88900(15)$ & $0.73061(9)$ & $0.0316(3)$ \\
\hline $\mathrm{O} 2$ & 0.48569 (13) & $0.88947(16)$ & $0.88653(10)$ & $0.0310(3)$ \\
\hline $\mathrm{O} 3$ & $0.68110(13)$ & $0.63211(16)$ & $0.71532(9)$ & $0.0275(3)$ \\
\hline $\mathrm{O} 4$ & $0.5610(2)$ & $0.6421(2)$ & $0.95580(11)$ & $0.0535(5)$ \\
\hline O5 & $0.68865(16)$ & 0.53978 (19) & $0.88111(12)$ & $0.0425(4)$ \\
\hline O6 & $0.7062(2)$ & $0.5199(3)$ & $1.00927(15)$ & $0.0780(8)$ \\
\hline O7 & $0.47104(13)$ & $0.45766(15)$ & $0.79809(10)$ & $0.0297(3)$ \\
\hline $\mathrm{O} 8$ & $0.27693(15)$ & $0.46317(18)$ & $0.76534(12)$ & $0.0430(4)$ \\
\hline O9 & $0.37120(17)$ & $0.26983(17)$ & $0.77663(12)$ & $0.0431(4)$ \\
\hline $\mathrm{O} 10$ & $0.51235(17)$ & $0.6218(2)$ & $0.38477(11)$ & $0.0446(4)$ \\
\hline O11 & $0.57284(17)$ & $0.6504(3)$ & $0.50502(13)$ & $0.0598(6)$ \\
\hline $\mathrm{O} 12$ & $0.38755(14)$ & $0.70400(18)$ & $0.47151(9)$ & $0.0337(4)$ \\
\hline N1 & $0.4200(2)$ & $0.6697(2)$ & $0.67879(12)$ & $0.0302(4)$ \\
\hline $\mathrm{H} 1 \mathrm{~A}$ & $0.3464(17)$ & $0.647(3)$ & $0.6859(16)$ & $0.040(8)^{*}$ \\
\hline H1B & $0.454(3)$ & $0.608(3)$ & $0.6519(16)$ & $0.050(9)^{*}$ \\
\hline N2 & $0.42249(18)$ & $0.7916(2)$ & $0.63406(11)$ & $0.0294(4)$ \\
\hline $\mathrm{H} 2$ & $0.401(3)$ & $0.789(3)$ & $0.5854(11)$ & $0.048(9)^{*}$ \\
\hline N3 & $0.31650(17)$ & $0.7017(2)$ & $0.86115(13)$ & $0.0301(4)$ \\
\hline $\mathrm{H} 3 \mathrm{~A}$ & $0.265(2)$ & $0.694(3)$ & $0.8222(13)$ & $0.038(7)^{*}$ \\
\hline $\mathrm{H} 3 \mathrm{~B}$ & $0.302(3)$ & $0.635(2)$ & $0.8919(16)$ & $0.051(9)^{*}$ \\
\hline N4 & $0.29181(17)$ & $0.8249(2)$ & $0.90180(12)$ & $0.0327(4)$ \\
\hline H4 & $0.2186(19)$ & $0.842(3)$ & $0.9187(18)$ & $0.060(10)^{*}$ \\
\hline N5 & $0.74028(16)$ & $0.82302(19)$ & $0.82323(11)$ & $0.0243(4)$ \\
\hline $\mathrm{H} 5 \mathrm{~A}$ & $0.786(2)$ & $0.809(3)$ & $0.8643(13)$ & $0.040(8)^{*}$ \\
\hline H5B & $0.722(2)$ & $0.9075(18)$ & $0.8197(16)$ & $0.040(8)^{*}$ \\
\hline N6 & $0.81190(16)$ & $0.79567(19)$ & $0.75554(12)$ & $0.0274(4)$ \\
\hline H6 & $0.8761(18)$ & $0.842(2)$ & $0.7489(15)$ & $0.035(7)^{*}$ \\
\hline N7 & $0.6533(2)$ & $0.5651(2)$ & 0.95054 (14) & $0.0433(5)$ \\
\hline N8 & $0.36977(17)$ & $0.39549(19)$ & $0.77997(11)$ & $0.0290(4)$ \\
\hline N9 & $0.49126(17)$ & $0.6579(2)$ & $0.45403(11)$ & $0.0286(4)$ \\
\hline $\mathrm{C} 1$ & 0.48401 (19) & $0.8957(2)$ & $0.66389(13)$ & $0.0258(4)$ \\
\hline $\mathrm{C} 2$ & $0.4922(3)$ & $1.0227(3)$ & $0.61533(16)$ & $0.0426(6)$ \\
\hline $\mathrm{H} 2 \mathrm{~A}$ & 0.472625 & 1.100447 & 0.647550 & $0.064 *$ \\
\hline H2B & 0.435281 & 1.017233 & 0.571491 & $0.064 *$ \\
\hline
\end{tabular}




\begin{tabular}{lllll}
$\mathrm{H} 2 \mathrm{C}$ & 0.573927 & 1.032232 & 0.595665 & $0.064^{*}$ \\
$\mathrm{C} 3$ & $0.38049(19)$ & $0.9150(2)$ & $0.90919(12)$ & $0.0246(4)$ \\
$\mathrm{C} 4$ & $0.3478(2)$ & $1.0492(3)$ & $0.94566(15)$ & $0.0376(5)$ \\
$\mathrm{H} 4 \mathrm{~A}$ & 0.401145 & 1.066733 & 0.990255 & $0.056^{*}$ \\
$\mathrm{H} 4 \mathrm{~B}$ & 0.264351 & 1.046395 & 0.963032 & $0.056^{*}$ \\
$\mathrm{H} 4 \mathrm{C}$ & 0.356757 & 1.120640 & 0.907122 & $0.056^{*}$ \\
$\mathrm{C} 5$ & $0.77501(18)$ & $0.7031(2)$ & $0.70400(13)$ & $0.0246(4)$ \\
$\mathrm{C} 6$ & $0.8488(2)$ & $0.6862(3)$ & $0.63059(16)$ & $0.0421(6)$ \\
$\mathrm{H} 6 \mathrm{~A}$ & 0.895336 & 0.603072 & 0.634062 & $0.063^{*}$ \\
$\mathrm{H} 6 \mathrm{~B}$ & 0.903604 & 0.762411 & 0.625384 & $0.063^{*}$ \\
$\mathrm{H} 6 \mathrm{C}$ & 0.794817 & 0.682378 & 0.585182 & $0.063^{*}$ \\
\hline
\end{tabular}

Atomic displacement parameters $\left(\AA^{2}\right)$

\begin{tabular}{|c|c|c|c|c|c|c|}
\hline & $U^{11}$ & $U^{22}$ & $U^{33}$ & $U^{12}$ & $U^{13}$ & $U^{23}$ \\
\hline $\mathrm{Tb} 1$ & $0.01473(4)$ & $0.01950(5)$ & $0.01956(5)$ & 0.00037 (4) & $-0.00101(3)$ & $-0.00128(4)$ \\
\hline $\mathrm{O} 1$ & $0.0418(9)$ & $0.0248(8)$ & $0.0279(8)$ & $-0.0019(7)$ & $-0.0130(7)$ & $0.0027(6)$ \\
\hline $\mathrm{O} 2$ & $0.0230(7)$ & $0.0316(8)$ & $0.0386(9)$ & $-0.0015(6)$ & $0.0073(6)$ & $-0.0121(7)$ \\
\hline $\mathrm{O} 3$ & $0.0225(7)$ & $0.0280(8)$ & $0.0321(8)$ & $-0.0051(6)$ & $0.0061(6)$ & $-0.0090(6)$ \\
\hline $\mathrm{O} 4$ & $0.0599(13)$ & $0.0684(13)$ & $0.0320(10)$ & $0.0158(11)$ & $-0.0029(9)$ & $0.0067(9)$ \\
\hline O5 & $0.0319(9)$ & $0.0424(10)$ & $0.0530(12)$ & $0.0057(8)$ & $-0.0068(8)$ & $0.0097(9)$ \\
\hline O6 & $0.0641(14)$ & $0.102(2)$ & $0.0668(15)$ & $-0.0119(14)$ & $-0.0300(12)$ & $0.0547(14)$ \\
\hline O7 & $0.0236(7)$ & $0.0240(7)$ & $0.0414(9)$ & $-0.0037(6)$ & $-0.0017(6)$ & $-0.0023(7)$ \\
\hline O8 & $0.0224(8)$ & $0.0387(10)$ & $0.0679(13)$ & $0.0003(7)$ & $-0.0027(8)$ & $0.0016(9)$ \\
\hline O9 & $0.0467(11)$ & $0.0227(8)$ & $0.0598(12)$ & $-0.0082(8)$ & $-0.0065(9)$ & $0.0001(8)$ \\
\hline $\mathrm{O} 10$ & $0.0487(11)$ & $0.0525(11)$ & $0.0331(9)$ & $0.0088(9)$ & $0.0142(8)$ & $-0.0051(8)$ \\
\hline O11 & $0.0280(9)$ & $0.1001(17)$ & $0.0510(12)$ & $0.0141(10)$ & $-0.0115(9)$ & $-0.0132(12)$ \\
\hline $\mathrm{O} 12$ & $0.0214(7)$ & $0.0512(10)$ & $0.0284(8)$ & $0.0049(7)$ & $0.0009(6)$ & $-0.0029(7)$ \\
\hline N1 & $0.0331(10)$ & $0.0294(10)$ & $0.0278(9)$ & $-0.0056(8)$ & $-0.0048(8)$ & $-0.0015(8)$ \\
\hline $\mathrm{N} 2$ & $0.0332(10)$ & $0.0370(11)$ & $0.0178(8)$ & $-0.0010(8)$ & $-0.0041(7)$ & $0.0008(8)$ \\
\hline N3 & $0.0217(8)$ & $0.0310(10)$ & $0.0377(11)$ & $-0.0035(8)$ & $0.0021(8)$ & $-0.0029(9)$ \\
\hline N4 & $0.0198(8)$ & $0.0376(11)$ & $0.0410(11)$ & $0.0027(8)$ & $0.0094(8)$ & $-0.0069(9)$ \\
\hline N5 & $0.0234(8)$ & $0.0233(9)$ & $0.0261(9)$ & $-0.0011(7)$ & $-0.0032(7)$ & $-0.0024(7)$ \\
\hline N6 & $0.0213(8)$ & $0.0257(9)$ & $0.0352(10)$ & $-0.0075(7)$ & $0.0036(7)$ & $-0.0022(8)$ \\
\hline N7 & 0.0389 (11) & $0.0437(13)$ & $0.0470(13)$ & $-0.0100(10)$ & $-0.0171(10)$ & $0.0220(11)$ \\
\hline N8 & $0.0277(9)$ & $0.0257(9)$ & $0.0337(10)$ & $-0.0065(8)$ & $0.0027(8)$ & $-0.0008(8)$ \\
\hline N9 & $0.0237(9)$ & $0.0321(10)$ & $0.0300(9)$ & $-0.0011(7)$ & $0.0041(7)$ & $0.0022(8)$ \\
\hline $\mathrm{C} 1$ & $0.0233(10)$ & $0.0299(11)$ & $0.0242(9)$ & $0.0054(8)$ & $0.0013(8)$ & $0.0019(8)$ \\
\hline $\mathrm{C} 2$ & $0.0429(14)$ & $0.0443(14)$ & 0.0404 (14) & $-0.0028(12)$ & $-0.0072(11)$ & $0.0157(12)$ \\
\hline $\mathrm{C} 3$ & $0.0248(9)$ & $0.0301(10)$ & $0.0189(9)$ & $0.0048(9)$ & $0.0016(7)$ & $-0.0013(8)$ \\
\hline $\mathrm{C} 4$ & $0.0361(12)$ & $0.0364(13)$ & $0.0403(13)$ & $0.0112(10)$ & $0.0027(10)$ & $-0.0104(11)$ \\
\hline $\mathrm{C} 5$ & $0.0221(9)$ & $0.0221(9)$ & $0.0296(10)$ & $0.0016(8)$ & $0.0035(8)$ & $0.0010(8)$ \\
\hline C6 & $0.0414(13)$ & $0.0411(14)$ & $0.0445(14)$ & $-0.0059(12)$ & $0.0206(11)$ & $-0.0097(12)$ \\
\hline
\end{tabular}

Geometric parameters $\left(\stackrel{A}{\circ}{ }^{\circ}\right)$

\begin{tabular}{llll}
\hline $\mathrm{Tb} 1-\mathrm{O} 1$ & $2.3632(15)$ & $\mathrm{N} 2-\mathrm{H} 2$ & $0.849(17)$ \\
$\mathrm{Tb} 1-\mathrm{O} 2$ & $2.3690(15)$ & $\mathrm{N} 2-\mathrm{C} 1$ & $1.316(3)$
\end{tabular}




\begin{tabular}{|c|c|c|c|}
\hline $\mathrm{Tb} 1-\mathrm{O} 3$ & $2.3525(14)$ & $\mathrm{N} 3-\mathrm{H} 3 \mathrm{~A}$ & 0.865 (17) \\
\hline $\mathrm{Tb} 1-\mathrm{O} 4$ & 2.4779 (19) & N3-H3B & $0.850(17)$ \\
\hline $\mathrm{Tb} 1-\mathrm{O} 5$ & 2.4959 (17) & $\mathrm{N} 3-\mathrm{N} 4$ & $1.414(3)$ \\
\hline $\mathrm{Tb} 1-\mathrm{O} 7$ & $2.4220(15)$ & $\mathrm{N} 4-\mathrm{H} 4$ & 0.867 (17) \\
\hline $\mathrm{Tb} 1-\mathrm{N} 1$ & $2.587(2)$ & $\mathrm{N} 4-\mathrm{C} 3$ & $1.313(3)$ \\
\hline $\mathrm{Tb} 1-\mathrm{N} 3$ & $2.5640(19)$ & N5-H5A & 0.857 (17) \\
\hline $\mathrm{Tb} 1-\mathrm{N} 5$ & $2.5532(18)$ & N5-H5B & 0.851 (17) \\
\hline $\mathrm{O} 1-\mathrm{C} 1$ & $1.240(2)$ & N5-N6 & $1.417(3)$ \\
\hline $\mathrm{O} 2-\mathrm{C} 3$ & $1.240(2)$ & N6-H6 & 0.845 (17) \\
\hline $\mathrm{O} 3-\mathrm{C} 5$ & $1.254(2)$ & N6-C5 & $1.314(3)$ \\
\hline $\mathrm{O} 4-\mathrm{N} 7$ & $1.261(3)$ & $\mathrm{C} 1-\mathrm{C} 2$ & $1.491(3)$ \\
\hline O5-N7 & $1.262(3)$ & $\mathrm{C} 2-\mathrm{H} 2 \mathrm{~A}$ & 0.9600 \\
\hline $\mathrm{O} 6-\mathrm{N} 7$ & $1.222(3)$ & $\mathrm{C} 2-\mathrm{H} 2 \mathrm{~B}$ & 0.9600 \\
\hline $\mathrm{O} 7-\mathrm{N} 8$ & $1.294(2)$ & $\mathrm{C} 2-\mathrm{H} 2 \mathrm{C}$ & 0.9600 \\
\hline $\mathrm{O} 8-\mathrm{N} 8$ & $1.232(2)$ & $\mathrm{C} 3-\mathrm{C} 4$ & $1.494(3)$ \\
\hline $\mathrm{O} 9-\mathrm{N} 8$ & $1.230(2)$ & $\mathrm{C} 4-\mathrm{H} 4 \mathrm{~A}$ & 0.9600 \\
\hline $\mathrm{O} 10-\mathrm{N} 9$ & $1.244(2)$ & $\mathrm{C} 4-\mathrm{H} 4 \mathrm{~B}$ & 0.9600 \\
\hline $\mathrm{O} 11-\mathrm{N} 9$ & $1.231(3)$ & $\mathrm{C} 4-\mathrm{H} 4 \mathrm{C}$ & 0.9600 \\
\hline $\mathrm{O} 12-\mathrm{N} 9$ & $1.257(2)$ & $\mathrm{C} 5-\mathrm{C} 6$ & $1.494(3)$ \\
\hline $\mathrm{N} 1-\mathrm{H} 1 \mathrm{~A}$ & 0.844 (17) & C6-H6A & 0.9600 \\
\hline $\mathrm{N} 1-\mathrm{H} 1 \mathrm{~B}$ & 0.845 (17) & C6-H6B & 0.9600 \\
\hline $\mathrm{N} 1-\mathrm{N} 2$ & $1.411(3)$ & $\mathrm{C} 6-\mathrm{H} 6 \mathrm{C}$ & 0.9600 \\
\hline $\mathrm{O} 1-\mathrm{Tb} 1-\mathrm{O} 2$ & $69.15(6)$ & $\mathrm{H} 3 \mathrm{~A}-\mathrm{N} 3-\mathrm{H} 3 \mathrm{~B}$ & $106(3)$ \\
\hline $\mathrm{O} 1-\mathrm{Tb} 1-\mathrm{O} 4$ & $137.09(7)$ & N4-N3-Tb1 & $111.81(13)$ \\
\hline $\mathrm{O} 1-\mathrm{Tb} 1-\mathrm{O} 5$ & $140.08(6)$ & $\mathrm{N} 4-\mathrm{N} 3-\mathrm{H} 3 \mathrm{~A}$ & $108.2(18)$ \\
\hline $\mathrm{O} 1-\mathrm{Tb} 1-\mathrm{O} 7$ & $135.12(5)$ & $\mathrm{N} 4-\mathrm{N} 3-\mathrm{H} 3 \mathrm{~B}$ & $109(2)$ \\
\hline $\mathrm{O} 1-\mathrm{Tb} 1-\mathrm{N} 1$ & $63.66(6)$ & N3-N4-H4 & $120(2)$ \\
\hline $\mathrm{O} 1-\mathrm{Tb} 1-\mathrm{N} 3$ & $98.35(6)$ & $\mathrm{C} 3-\mathrm{N} 4-\mathrm{N} 3$ & $118.22(18)$ \\
\hline $\mathrm{O} 1-\mathrm{Tb} 1-\mathrm{N} 5$ & $69.45(6)$ & $\mathrm{C} 3-\mathrm{N} 4-\mathrm{H} 4$ & $121(2)$ \\
\hline $\mathrm{O} 2-\mathrm{Tb} 1-\mathrm{O} 4$ & $70.66(7)$ & Tb1-N5-H5A & $118.0(19)$ \\
\hline $\mathrm{O} 2-\mathrm{Tb} 1-\mathrm{O} 5$ & $113.77(6)$ & $\mathrm{Tb} 1-\mathrm{N} 5-\mathrm{H} 5 \mathrm{~B}$ & 106.4 (19) \\
\hline $\mathrm{O} 2-\mathrm{Tb} 1-\mathrm{O} 7$ & $138.48(5)$ & $\mathrm{H} 5 \mathrm{~A}-\mathrm{N} 5-\mathrm{H} 5 \mathrm{~B}$ & $110(3)$ \\
\hline $\mathrm{O} 2-\mathrm{Tb} 1-\mathrm{N} 1$ & 114.19 (6) & $\mathrm{N} 6-\mathrm{N} 5-\mathrm{Tb} 1$ & $109.48(12)$ \\
\hline $\mathrm{O} 2-\mathrm{Tb} 1-\mathrm{N} 3$ & $64.41(6)$ & $\mathrm{N} 6-\mathrm{N} 5-\mathrm{H} 5 \mathrm{~A}$ & $107.6(19)$ \\
\hline $\mathrm{O} 2-\mathrm{Tb} 1-\mathrm{N} 5$ & $76.73(5)$ & $\mathrm{N} 6-\mathrm{N} 5-\mathrm{H} 5 \mathrm{~B}$ & 104.8 (19) \\
\hline $\mathrm{O} 3-\mathrm{Tb} 1-\mathrm{O} 1$ & $79.00(6)$ & N5-N6-H6 & $118.1(18)$ \\
\hline $\mathrm{O} 3-\mathrm{Tb} 1-\mathrm{O} 2$ & $137.59(5)$ & $\mathrm{C} 5-\mathrm{N} 6-\mathrm{N} 5$ & $119.68(17)$ \\
\hline $\mathrm{O} 3-\mathrm{Tb} 1-\mathrm{O} 4$ & $124.67(6)$ & $\mathrm{C} 5-\mathrm{N} 6-\mathrm{H} 6$ & $122.1(18)$ \\
\hline $\mathrm{O} 3-\mathrm{Tb} 1-\mathrm{O} 5$ & $74.51(6)$ & $\mathrm{O} 4-\mathrm{N} 7-\mathrm{O} 5$ & 115.89 (19) \\
\hline $\mathrm{O} 3-\mathrm{Tb} 1-\mathrm{O} 7$ & $83.93(5)$ & $\mathrm{O} 6-\mathrm{N} 7-\mathrm{O} 4$ & $121.8(3)$ \\
\hline $\mathrm{O} 3-\mathrm{Tb} 1-\mathrm{N} 1$ & $72.54(6)$ & $\mathrm{O} 6-\mathrm{N} 7-\mathrm{O} 5$ & $122.3(3)$ \\
\hline $\mathrm{O} 3-\mathrm{Tb} 1-\mathrm{N} 3$ & $150.36(6)$ & $\mathrm{O} 8-\mathrm{N} 8-\mathrm{O} 7$ & $119.45(18)$ \\
\hline $\mathrm{O} 3-\mathrm{Tb} 1-\mathrm{N} 5$ & $66.06(5)$ & $\mathrm{O} 9-\mathrm{N} 8-\mathrm{O} 7$ & $117.96(19)$ \\
\hline $\mathrm{O} 4-\mathrm{Tb} 1-\mathrm{O} 5$ & $50.93(7)$ & $\mathrm{O} 9-\mathrm{N} 8-\mathrm{O} 8$ & $122.57(19)$ \\
\hline $\mathrm{O} 4-\mathrm{Tb} 1-\mathrm{N} 1$ & 150.97 (7) & $\mathrm{O} 10-\mathrm{N} 9-\mathrm{O} 12$ & 120.03 (19) \\
\hline $\mathrm{O} 4-\mathrm{Tb} 1-\mathrm{N} 3$ & $77.11(7)$ & $\mathrm{O} 11-\mathrm{N} 9-\mathrm{O} 10$ & $119.8(2)$ \\
\hline $\mathrm{O} 4-\mathrm{Tb} 1-\mathrm{N} 5$ & $87.34(7)$ & $\mathrm{O} 11-\mathrm{N} 9-\mathrm{O} 12$ & $120.1(2)$ \\
\hline
\end{tabular}




\begin{tabular}{|c|c|c|c|}
\hline $\mathrm{O} 5-\mathrm{Tb} 1-\mathrm{N} 1$ & $131.95(7)$ & $\mathrm{O} 1-\mathrm{C} 1-\mathrm{N} 2$ & $121.1(2)$ \\
\hline $\mathrm{O} 5-\mathrm{Tb} 1-\mathrm{N} 3$ & $119.27(7)$ & $\mathrm{O} 1-\mathrm{C} 1-\mathrm{C} 2$ & $120.9(2)$ \\
\hline $\mathrm{O} 5-\mathrm{Tb} 1-\mathrm{N} 5$ & $72.69(6)$ & $\mathrm{N} 2-\mathrm{C} 1-\mathrm{C} 2$ & $118.0(2)$ \\
\hline $\mathrm{O} 7-\mathrm{Tb} 1-\mathrm{O} 4$ & $86.19(7)$ & $\mathrm{C} 1-\mathrm{C} 2-\mathrm{H} 2 \mathrm{~A}$ & 109.5 \\
\hline $\mathrm{O} 7-\mathrm{Tb} 1-\mathrm{O} 5$ & $70.93(6)$ & $\mathrm{C} 1-\mathrm{C} 2-\mathrm{H} 2 \mathrm{~B}$ & 109.5 \\
\hline $\mathrm{O} 7-\mathrm{Tb} 1-\mathrm{N} 1$ & $71.68(6)$ & $\mathrm{C} 1-\mathrm{C} 2-\mathrm{H} 2 \mathrm{C}$ & 109.5 \\
\hline $\mathrm{O} 7-\mathrm{Tb} 1-\mathrm{N} 3$ & $77.33(6)$ & $\mathrm{H} 2 \mathrm{~A}-\mathrm{C} 2-\mathrm{H} 2 \mathrm{~B}$ & 109.5 \\
\hline $\mathrm{O} 7-\mathrm{Tb} 1-\mathrm{N} 5$ & $137.71(5)$ & $\mathrm{H} 2 \mathrm{~A}-\mathrm{C} 2-\mathrm{H} 2 \mathrm{C}$ & 109.5 \\
\hline $\mathrm{N} 3-\mathrm{Tb} 1-\mathrm{N} 1$ & $79.81(7)$ & $\mathrm{H} 2 \mathrm{~B}-\mathrm{C} 2-\mathrm{H} 2 \mathrm{C}$ & 109.5 \\
\hline $\mathrm{N} 5-\mathrm{Tb} 1-\mathrm{N} 1$ & $121.66(6)$ & $\mathrm{O} 2-\mathrm{C} 3-\mathrm{N} 4$ & $121.3(2)$ \\
\hline $\mathrm{N} 5-\mathrm{Tb} 1-\mathrm{N} 3$ & $140.98(6)$ & $\mathrm{O} 2-\mathrm{C} 3-\mathrm{C} 4$ & $122.1(2)$ \\
\hline $\mathrm{C} 1-\mathrm{O} 1-\mathrm{Tb} 1$ & $125.30(14)$ & $\mathrm{N} 4-\mathrm{C} 3-\mathrm{C} 4$ & 116.64 (19) \\
\hline $\mathrm{C} 3-\mathrm{O} 2-\mathrm{Tb} 1$ & $123.84(14)$ & $\mathrm{C} 3-\mathrm{C} 4-\mathrm{H} 4 \mathrm{~A}$ & 109.5 \\
\hline $\mathrm{C} 5-\mathrm{O} 3-\mathrm{Tb} 1$ & $121.19(13)$ & $\mathrm{C} 3-\mathrm{C} 4-\mathrm{H} 4 \mathrm{~B}$ & 109.5 \\
\hline $\mathrm{N} 7-\mathrm{O} 4-\mathrm{Tb} 1$ & $96.95(15)$ & $\mathrm{C} 3-\mathrm{C} 4-\mathrm{H} 4 \mathrm{C}$ & 109.5 \\
\hline $\mathrm{N} 7-\mathrm{O} 5-\mathrm{Tb} 1$ & $96.06(14)$ & $\mathrm{H} 4 \mathrm{~A}-\mathrm{C} 4-\mathrm{H} 4 \mathrm{~B}$ & 109.5 \\
\hline $\mathrm{N} 8-\mathrm{O} 7-\mathrm{Tb} 1$ & $136.41(13)$ & $\mathrm{H} 4 \mathrm{~A}-\mathrm{C} 4-\mathrm{H} 4 \mathrm{C}$ & 109.5 \\
\hline $\mathrm{Tb} 1-\mathrm{N} 1-\mathrm{H} 1 \mathrm{~A}$ & $111.2(19)$ & $\mathrm{H} 4 \mathrm{~B}-\mathrm{C} 4-\mathrm{H} 4 \mathrm{C}$ & 109.5 \\
\hline $\mathrm{Tb} 1-\mathrm{N} 1-\mathrm{H} 1 \mathrm{~B}$ & $108(2)$ & $\mathrm{O} 3-\mathrm{C} 5-\mathrm{N} 6$ & $121.60(19)$ \\
\hline $\mathrm{H} 1 \mathrm{~A}-\mathrm{N} 1-\mathrm{H} 1 \mathrm{~B}$ & $108(3)$ & $\mathrm{O} 3-\mathrm{C} 5-\mathrm{C} 6$ & $121.0(2)$ \\
\hline $\mathrm{N} 2-\mathrm{N} 1-\mathrm{Tb} 1$ & $112.25(13)$ & N6-C5-C6 & 117.39 (19) \\
\hline $\mathrm{N} 2-\mathrm{N} 1-\mathrm{H} 1 \mathrm{~A}$ & $109.1(19)$ & $\mathrm{C} 5-\mathrm{C} 6-\mathrm{H} 6 \mathrm{~A}$ & 109.5 \\
\hline $\mathrm{N} 2-\mathrm{N} 1-\mathrm{H} 1 \mathrm{~B}$ & $108(2)$ & $\mathrm{C} 5-\mathrm{C} 6-\mathrm{H} 6 \mathrm{~B}$ & 109.5 \\
\hline $\mathrm{N} 1-\mathrm{N} 2-\mathrm{H} 2$ & $119(2)$ & $\mathrm{C} 5-\mathrm{C} 6-\mathrm{H} 6 \mathrm{C}$ & 109.5 \\
\hline $\mathrm{C} 1-\mathrm{N} 2-\mathrm{N} 1$ & $117.60(18)$ & $\mathrm{H} 6 \mathrm{~A}-\mathrm{C} 6-\mathrm{H} 6 \mathrm{~B}$ & 109.5 \\
\hline $\mathrm{C} 1-\mathrm{N} 2-\mathrm{H} 2$ & $122(2)$ & $\mathrm{H} 6 \mathrm{~A}-\mathrm{C} 6-\mathrm{H} 6 \mathrm{C}$ & 109.5 \\
\hline $\mathrm{Tb} 1-\mathrm{N} 3-\mathrm{H} 3 \mathrm{~A}$ & $111.3(19)$ & $\mathrm{H} 6 \mathrm{~B}-\mathrm{C} 6-\mathrm{H} 6 \mathrm{C}$ & 109.5 \\
\hline $\mathrm{Tb} 1-\mathrm{N} 3-\mathrm{H} 3 \mathrm{~B}$ & $110(2)$ & & \\
\hline $\mathrm{Tb} 1-\mathrm{O} 1-\mathrm{C} 1-\mathrm{N} 2$ & $3.9(3)$ & $\mathrm{Tb} 1-\mathrm{O} 7-\mathrm{N} 8-\mathrm{O} 9$ & $178.18(15)$ \\
\hline $\mathrm{Tb} 1-\mathrm{O} 1-\mathrm{C} 1-\mathrm{C} 2$ & $-176.58(17)$ & $\mathrm{Tb} 1-\mathrm{N} 1-\mathrm{N} 2-\mathrm{C} 1$ & $1.4(2)$ \\
\hline $\mathrm{Tb} 1-\mathrm{O} 2-\mathrm{C} 3-\mathrm{N} 4$ & $8.5(3)$ & $\mathrm{Tb} 1-\mathrm{N} 3-\mathrm{N} 4-\mathrm{C} 3$ & $0.0(3)$ \\
\hline $\mathrm{Tb} 1-\mathrm{O} 2-\mathrm{C} 3-\mathrm{C} 4$ & $-171.10(16)$ & $\mathrm{Tb} 1-\mathrm{N} 5-\mathrm{N} 6-\mathrm{C} 5$ & $-6.9(2)$ \\
\hline $\mathrm{Tb} 1-\mathrm{O} 3-\mathrm{C} 5-\mathrm{N} 6$ & $14.8(3)$ & $\mathrm{N} 1-\mathrm{N} 2-\mathrm{C} 1-\mathrm{O} 1$ & $-3.4(3)$ \\
\hline $\mathrm{Tb} 1-\mathrm{O} 3-\mathrm{C} 5-\mathrm{C} 6$ & $-164.56(17)$ & $\mathrm{N} 1-\mathrm{N} 2-\mathrm{C} 1-\mathrm{C} 2$ & $177.1(2)$ \\
\hline $\mathrm{Tb} 1-\mathrm{O} 4-\mathrm{N} 7-\mathrm{O} 5$ & $-4.1(2)$ & $\mathrm{N} 3-\mathrm{N} 4-\mathrm{C} 3-\mathrm{O} 2$ & $-5.3(3)$ \\
\hline $\mathrm{Tb} 1-\mathrm{O} 4-\mathrm{N} 7-\mathrm{O} 6$ & $174.7(2)$ & $\mathrm{N} 3-\mathrm{N} 4-\mathrm{C} 3-\mathrm{C} 4$ & $174.4(2)$ \\
\hline $\mathrm{Tb} 1-\mathrm{O} 5-\mathrm{N} 7-\mathrm{O} 4$ & $4.0(2)$ & $\mathrm{N} 5-\mathrm{N} 6-\mathrm{C} 5-\mathrm{O} 3$ & $-4.1(3)$ \\
\hline $\mathrm{Tb} 1-\mathrm{O} 5-\mathrm{N} 7-\mathrm{O} 6$ & $-174.7(2)$ & $\mathrm{N} 5-\mathrm{N} 6-\mathrm{C} 5-\mathrm{C} 6$ & $175.3(2)$ \\
\hline $\mathrm{Tb} 1-\mathrm{O} 7-\mathrm{N} 8-\mathrm{O} 8$ & $-0.5(3)$ & & \\
\hline
\end{tabular}

Hydrogen-bond geometry $\left(A,{ }^{\circ}\right)$

\begin{tabular}{lllll}
\hline$D-\mathrm{H} \cdots A$ & $D-\mathrm{H}$ & $\mathrm{H} \cdots A$ & $D \cdots A$ & $D-\mathrm{H} \cdots A$ \\
\hline $\mathrm{N} 1-\mathrm{H} 1 A \cdots \mathrm{O} 8$ & $0.84(2)$ & $2.37(2)$ & $2.950(3)$ & $126(2)$ \\
$\mathrm{N} 1-\mathrm{H} 1 B \cdots \mathrm{O} 10^{\mathrm{i}}$ & $0.85(2)$ & $2.36(2)$ & $3.136(3)$ & $153(3)$ \\
$\mathrm{N} 2-\mathrm{H} 2 \cdots \mathrm{O} 11$ & $0.85(2)$ & $2.69(3)$ & $3.070(3)$ & $109(2)$ \\
$\mathrm{N} 2-\mathrm{H} 2 \cdots \mathrm{O} 12$ & $0.85(2)$ & $2.09(2)$ & $2.891(2)$ & $156(3)$
\end{tabular}


supporting information

\begin{tabular}{lllll}
$\mathrm{N} 3-\mathrm{H} 3 A \cdots \mathrm{O} 8$ & $0.87(2)$ & $2.46(3)$ & $2.866(3)$ & $110(2)$ \\
$\mathrm{N} 3-\mathrm{H} 3 A \cdots \mathrm{O} 9^{\text {ii }}$ & $0.87(2)$ & $2.33(2)$ & $3.146(3)$ & $157(2)$ \\
$\mathrm{N} 3-\mathrm{H} 3 B \cdots \mathrm{O} 6^{\text {iii }}$ & $0.85(2)$ & $2.25(2)$ & $3.089(3)$ & $168(3)$ \\
$\mathrm{N} 4-\mathrm{H} 4 \cdots \mathrm{O} 10^{\text {iv }}$ & $0.87(2)$ & $2.34(2)$ & $3.102(3)$ & $147(3)$ \\
$\mathrm{N} 4-\mathrm{H} 4 \cdots \mathrm{O} 11^{\text {iv }}$ & $0.87(2)$ & $2.17(2)$ & $2.984(3)$ & $156(3)$ \\
$\mathrm{N} 5-\mathrm{H} 5 A \cdots \mathrm{O} 10^{\mathrm{v}}$ & $0.86(2)$ & $2.58(2)$ & $3.176(3)$ & $128(2)$ \\
$\mathrm{N} 5-\mathrm{H} 5 A \cdots \mathrm{O} 12^{\mathrm{v}}$ & $0.86(2)$ & $2.11(2)$ & $2.964(2)$ & $173(3)$ \\
$\mathrm{N} 5-\mathrm{H} 5 B \cdots \mathrm{O} 3^{\mathrm{vi}}$ & $0.85(2)$ & $2.51(2)$ & $3.211(2)$ & $140(2)$ \\
$\mathrm{N} 6-\mathrm{H} 6 \cdots \mathrm{O} 7^{\mathrm{vi}}$ & $0.85(2)$ & $2.17(2)$ & $2.999(2)$ & $166(2)$ \\
$\mathrm{N} 6-\mathrm{H} 6 \cdots \mathrm{O} 10^{\mathrm{v}}$ & $0.85(2)$ & $2.74(2)$ & $3.170(3)$ & $114(2)$ \\
\hline
\end{tabular}

Symmetry codes: (i) $-x+1,-y+1,-z+1$; (ii) $-x+1 / 2, y+1 / 2,-z+3 / 2$; (iii) $-x+1,-y+1,-z+2$; (iv) $x-1 / 2,-y+3 / 2, z+1 / 2$; (v) $x+1 / 2,-y+3 / 2, z+1 / 2$; (vi) $-x+3 / 2, y+1 / 2,-z+3 / 2$. 